Economía, Sociedad y Territorio, vol. vIII, núm. 27, 2008, 721-738

\title{
El turismo en el espacio rural en la mitad sur de Río Grande do Sul, Brasil (1997-2005)
}

\section{Tourism in the rural space in Southern Río Grande do Sul, Brazil (1997-2005)}

\author{
EURICO DE OliveIRA-SANTOS \\ Carlos Ernesto González-Esquivel*
}

\begin{abstract}
In this paper, we have studied nine rural estates in Southern Rio Grande do Sul (Brazil) in order to evaluate their evolution and to differentiate those dedicated to agro-tourism from those that offer rural tourism. This study was carried out in two parts; an exploratory qualitative part and a descriptive quantitative one that included surveys in three different times: 1997, 2002 and 2005. The results allow us to conclude that multi-activity is the most wide spread strategy in order to face macroeconomic conditions. We also noticed that agro-tourism is more predominant than rural tourism. This study shows the viability of tourist activities in some estates while generating additional income and allowing the contact with urban in habitants.
\end{abstract}

Keywords: rural tourism, agro-tourism, Rio Grande do Sul, Brazil.

\section{Resumen}

Con el objetivo de analizar la evolución de las propiedades rurales, así como diferenciar las que practican agroturismo de las que ofrecen turismo rural, se investigaron nueve propiedades rurales en la mitad sur del estado de Rio Grande do Sul, Brasil. El estudio se llevó a cabo en dos fases: una cualitativa con carácter exploratorio y otra cuantitativa con carácter descriptivo que consistió en la aplicación de encuestas en tres momentos diferentes: 1997, 2002 y 2005. Los resultados permiten concluir que la pluriactividad es la estrategia más adoptada para enfrentar las condiciones macroeconómicas; asimismo, se detectó predominancia del agroturismo sobre el turismo rural. El estudio mostró la viabilidad de las actividades turísticas en algunas fincas, toda vez que generan ingresos adicionales y permiten el contacto con los habitantes urbanos.

Palabras clave: turismo rural, agroturismo, Rio Grande do Sul, Brasil.

* Universidad Autónoma del Estado de México. Correo-e: eurico58@terra.com.br, cge1@uaemex.mx. 


\section{Introducción}

Considerando la crisis que muchos propietarios rurales enfrentan por la disminución de los precios de los productos agropecuarios, es importante que se desarrolle la idea de que un ingreso suplementario que surja, por ejemplo, del agroturismo o del turismo rural puede ser una opción viable (Veiga, 2002). Fabíola Zdepski también refiere que "el turismo rural surge como alternativa para que los propietarios enfrenten la actual crisis, debido a la falta de incentivo para las actividades agropecuarias" (2001: 156). El agroturismo, de acuerdo con la Associazione per il Turismo in Ambiente Rurale (ATAR, 1984), más que una fuente de renta adicional funciona como medio de desarrollo sociocultural a través de una nueva relación urbano-rural que involucra una multiplicidad de valores (humanos, sociales, culturales, etc.). Según José Veiga (2002), los países desarrollados están en un proceso de vuelta a las riquezas naturales donde los encantos de la vida rural -belleza, tranquilidad y seguridad- han sido muy valorados, en especial por habitantes rurales, turistas y algunos tipos de empresarios. Para José María Carneiro y Renato Maluf "una fórmula con profundas raíces en la experiencia europea no puede ser trasladada mecánicamente a la realidad brasileña, entre otras razones, por el papel central que se atribuye a la noción de multifuncionalidad" (2003: 137). El agroturismo y el turismo rural forman parte de la multifuncionalidad del medio rural brasileño. Con el fin de mejorar el éxito de la relación urbano-rural, algunos propietarios han tomado medidas para crear establecimientos ubicados en zonas rurales que ofrecen algunas comodidades urbanas, con la intención de proporcionar momentos agradables con los atractivos del medio rural. El agroturismo y el turismo rural han tenido un éxito relativo en diversas partes del mundo. Por ejemplo, Gemma Cánoves et al. (2004) señalan que el turismo rural se popularizó en Europa desde los sesenta y se ha convertido en una fuente de ingreso complementario para entre 2 y $20 \%$ de las fincas en diferentes países. Aunque el modelo no sea homogéneo, un denominador común es el contacto directo entre el turista y los propietarios. De acuerdo con estos autores, el número de fincas en España que reciben turistas creció más de seis veces entre 1994 y 2003.

Varios teóricos del tema definen al agroturismo como una modalidad del turismo rural, que se caracteriza por la visita a empresas rurales para conocer y practicar las actividades que se 
desarrollan ahí. Esto puede originar un conjunto de actividades asociadas como hotel-hacienda, pesca y paga, posada, restaurante típico, venta directa de productos locales, artesanía, industrialización y otras actividades de recreación que tienen que ver con la vida cotidiana y con los trabajos del campo (Riveros y Blanco, 2003). También es relevante el concepto de Clayton Campanhola y José Graziano acerca del agroturismo:

Actividades internas de la propiedad, que generan ocupaciones complementarias a las actividades agrícolas, las cuales siguen haciendo parte de lo cotidiano de la propiedad, en menor o mayor intensidad, que deben ser entendidas como parte de un proceso de agregación de servicios a los productos agrícolas y bienes no materiales existentes en las propiedades rurales (paisajes, aire puro, etc.), a partir del tiempo libre de las familias agrícolas con eventuales contrataciones de mano de obra externa (2003: 148).

De acuerdo con Luis Andrés Martínez (2005), el turismo rural se define por el ámbito en que los turistas desarrollan sus actividades más que por el contenido de las tareas que realizan. De esta manera, la base de este tipo de turismo es el medio rural en un sentido amplio, el cual posee un interesante patrimonio natural, histórico-cultural y arquitectónico que despierta el interés de las personas que habitualmente viven en el medio urbano y que están dispuestas a invertir parte de su tiempo libre y recursos en reencontrarse, en muchos casos, con sus orígenes o simplemente buscan formas o lugares para vacacionar que se encuentran más allá de su tipo de vida habitual.

Para los autores brasileños, entre ellos José Graziano et al. (citados por Tulik, 2003), es más adecuado referirse a la totalidad de los movimientos turísticos que se desarrollan en el medio rural con las expresiones turismo en el espacio rural o turismo en las áreas rurales, por lo que proponen, entonces, que el término turismo rural se reserve para aquellas actividades que, en mayor grado, se identifican con las especificidades de la vida rural.

Casio Oliveira (2001) afirma que la disminución de inversión en actividades primarias lleva a una baja de la productividad y del ingreso, con la consecuente reducción de posibles atractivos turísticos, ya que las actividades agropecuarias pueden ser importantes insumos para el agroturismo y el turismo rural. Debido a su multifuncionalidad como actividades generadoras de empleos e ingreso para promover las infraestructuras de intercambios entre los medios rural y urbano, el agroturismo y el tu- 
rismo rural pueden generar desarrollo en regiones menos favorecidas. Estas actividades todavía no están pensadas para recibir a muchas personas y tampoco son una solución definitiva a los graves problemas del sector primario, pero pueden convertirse en una renta adicional para los productores. Es posible que esto contribuya a un desarrollo sustentable, lo que implica una mejoría en la calidad de vida rural, respetando la capacidad de soporte del ecosistema con la participación del ciudadano urbano e involucrando los valores socioculturales de ambos.

El objetivo general de este estudio es evaluar el desarrollo de la actividad turística en nueve propiedades rurales de la mitad sur del estado de Río Grande do Sul, Brasil.

Para eso tenemos que ubicarnos en el espacio. En lo que se refiere a la familia, para que tengamos una noción de su perfil en cuanto a los aspectos de escolaridad y número de miembros, es necesario conocer su historia para saber si esta finca pertenece hace mucho tiempo a esta familia y quién dirige la empresa, así como saber qué porcentajes representan tanto las actividades primarias como las terciarias.

Específicamente, se buscó diferenciar en qué propiedades se practica agroturismo y en cuáles turismo rural, así como caracterizar, en un primer acercamiento, estas fincas con actividades turísticas en relación con los siguientes aspectos: localización, datos familiares, historia y administración, actividad primaria, infraestructura y actividad turística. Este artículo tiene como tema principal el desarrollo del agroturismo y del turismo rural -de manera que el turista no es el protagonista del estudio- y se basa en el trinomio propiedad, propietarios y generación de empleo y renta.

Se eligió al propietario rural como foco principal de la investigación y quedó en segundo lugar la perspectiva del turista propiamente dicho, lo cual no atribuye mayor o menor valor a ninguna de las perspectivas, ya que ambas tienen su importancia dentro del agroturismo y del turismo rural, pero en actividades no agrícolas hay un mayor grado de afinidad con el propietario rural/ciencias agrarias que con el turista/turismo.

Partimos de la hipótesis de que el agroturismo y el turismo rural constituyen opciones de renta complementaria para las propiedades de la mitad sur de Río Grande do Sul. Esto lleva a una evolución dirigida al carácter pluriactivo, es decir, a la estrategia que las familias rurales han tomado para adaptarse a las condiciones macroeconómicas sin perder la esencia del medio rural, basada en la agricultura y la ganadería. Se hizo énfasis en la pers- 
pectiva del propietario sin descartar que en estudios posteriores se pueda realizar un análisis de la demanda.

\section{Metodología}

Se hizo un estudio longitudinal de las propiedades rurales de la mitad sur del estado de Río Grande do Sul, Brasil, de acuerdo con la información del gobierno estatal (Setur, 1997). Para Claire Seltiz et al. (1987), una forma de clasificación de los tipos de investigación es la que considera la relación con el tiempo, ya que el investigador puede tener interés en estudiar una determinada variable y sus efectos a lo largo del tiempo.

En total se identificaron nueve propiedades en las cuales se practica el agroturismo o el turismo rural, lo que indica lo reciente de la actividad en la región. Para evaluar el desarrollo de la actividad turística y de las fincas en general, entrevistamos a los propietarios en tres etapas diferentes, febrero de 1997, julio de 2002 y marzo de 2005 con el fin de verificar la evolución de la actividad en un periodo amplio.

Las encuestas fueron del tipo semiestructurado e incluyeron preguntas abiertas y cerradas. Las respuestas a las preguntas cerradas se tabularon y analizaron por categorías y frecuencias. Las preguntas abiertas se sometieron a análisis de contenido (Bardin, 1977).

\section{Resultados y discusión}

El análisis de los resultados se presenta en cinco partes: localización de las propiedades; características de los propietarios e historia de las propiedades; actividad turística; actividad primaria y generación de empleo y, por último, renta por sector. Consideramos que estos aspectos abordan de manera amplia las variables del objetivo de este estudio.

\section{Localización y tamaño de las propiedades}

Sólo se identificaron nueve fincas que practicaban el agroturismo o el turismo rural en 1997, lo que indica lo reciente de la actividad en la región. La mayor parte de las propiedades se ubica en las regiones de Campanha (cinco) y Centro-Sul (tres). Cabe hacer notar que las cinco fincas de la primera región actualmente se encuentran cerradas. La superficie de las propiedades va de 
789 ha a 2,100 ha, con una media de 1,185.2 ha (d.e. 363.9 ha). Según datos de Engevix (1997), la superficie media de las propiedades rurales de la mitad sur es menor de 50 ha, por lo que al momento de la primera encuesta sólo grandes propietarios habían incursionado en la actividad.

La distancia entre la capital del estado, Porto Alegre, y las fincas va de 125 a $498 \mathrm{~km}$, con la mayor parte de las propiedades en el rango de 300 a $400 \mathrm{~km}$. Debido al cierre de algunas de ellas, la distancia promedio en las fincas que todavía funcionaban en 2005 se redujo a un rango de 125 a $140 \mathrm{~km}$ de la capital. Esto coincide con lo que refiere Adonis Zimmermann (1996) en el sentido de que el agroturismo y el turismo rural regional tienen su mayor clientela en un radio de $150 \mathrm{~km}$, es decir, la distancia pudo ser uno de los factores del cierre de estas actividades en el periodo de estudio. Francisco Martínez y Javier Monzonís (s.f.) proponen una clasificación del tiempo de estancia en relación con la distancia, según la cual cuando el tiempo de viaje es de una hora y media y la distancia menor a $150 \mathrm{~km}$, generalmente los huéspedes se quedan el fin de semana; cuando la distancia va de 150 a $300 \mathrm{~km}$, se quedan en días festivos y cuando la distancia es mayor, en el periodo de vacaciones.

Dichos autores corroboraron su clasificación en la realidad: las distancias de la capital del estado a las propiedades son muy grandes y la falta de transporte de buena calidad tanto carretero como aéreo aún es precario en esa región, por lo que, básicamente, el agroturismo y el turismo rural son una actividad de proximidad.

\section{Características de los propietarios e historia de las propiedades}

En cuanto al género de los propietarios rurales de los establecimientos activos investigados, seis son hombres y tres mujeres. En algunas ocasiones la mujer se convierte en propietaria rural por muerte de sus padres o su marido, es decir, reciben las fincas como herencia. Pudimos observar propietarias que mantienen afanosamente estas empresas. Asimismo, en varios casos la mujer participa más que el hombre en la actividad turística, mientras que éste permanece en las actividades primarias. La mitad de los encuestados cuenta con estudios de tercer grado (bachillerato) o superiores, tanto en ciencias agropecuarias como en otras áreas. Casi ninguno tiene sólo educación básica, por lo que 


\section{Mapa I}

Localización de la mitad sur del estado de Río Grande do Sul, Brasil

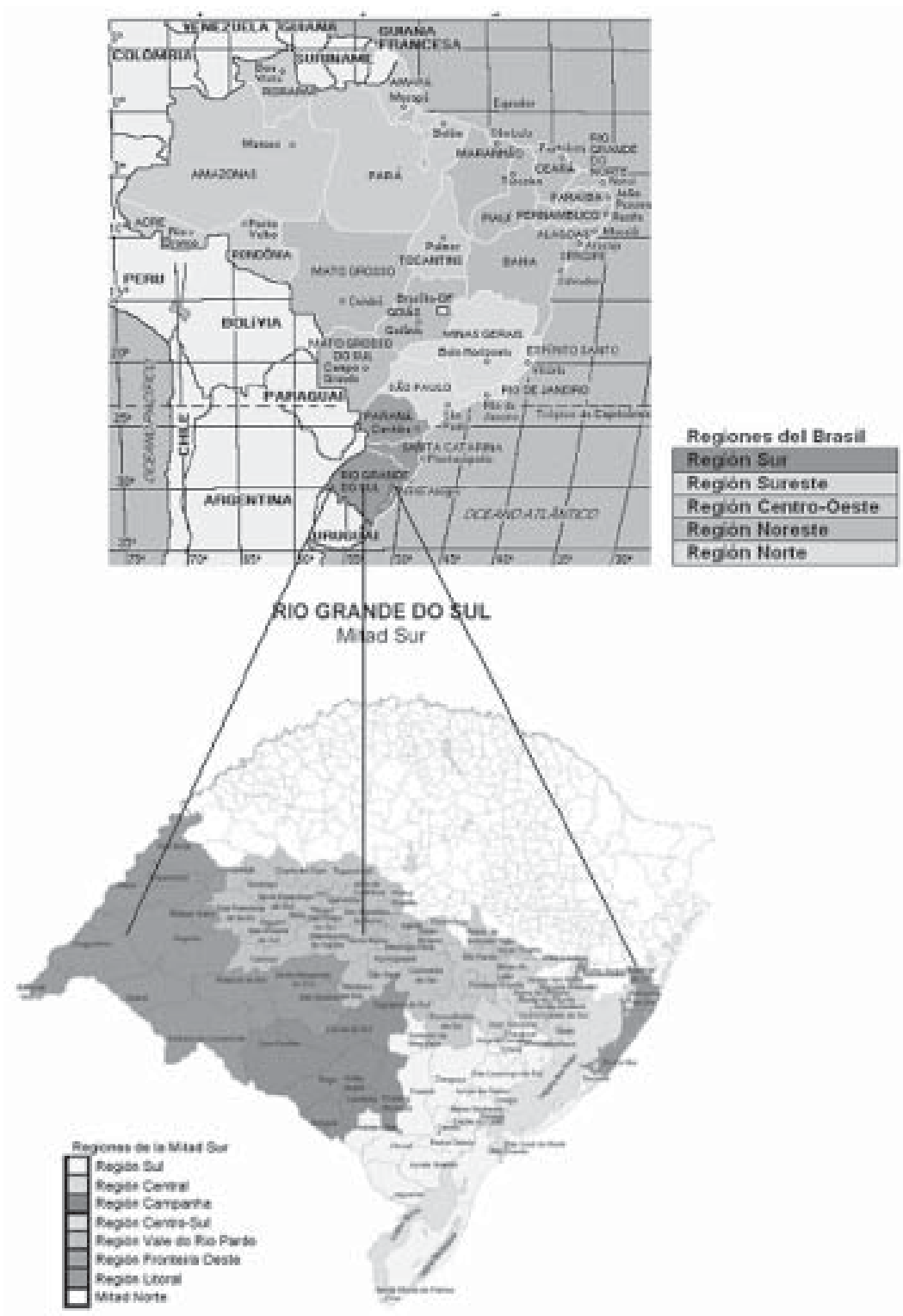

Fuente: http://www.guianet.com.br/guiacidades/ y http://www.setur.rs.gov.br. 
se considera que el nivel de escolaridad es alto. Un aspecto interesante es que ninguno de los dueños vive en la propiedad. Dos viven en Porto Alegre y el resto en ciudades del interior del estado. Según José Veiga, "un indicador del carácter multisectorial de la renta de los agricultores es el lugar de residencia” (2002: 111). El censo agropecuario 1995-1996 mostró la enorme frecuencia con que los agricultores (propietarios rurales) no viven en el establecimiento, sino en zona urbana. La escolaridad de los hijos es alta y destaca que, de quienes poseen estudios universitarios, $72.7 \%$ son en áreas no agropecuarias, es decir, hay poco interés por las actividades del campo. Sólo 36.4\% de los hijos viven en la propiedad y normalmente son aquellos que no continuaron sus estudios después de la educación media superior. Los datos dejan dudas sobre la permanencia de las familias en las propiedades durante las próximas generaciones.

En cuanto a la historia de la propiedad en la familia, existe una división lineal: dos vienen desde la sexta generación, dos desde la quinta, tres desde la cuarta y tres desde la tercera. Esto parece indicar un apego de las familias por su patrimonio, pero al mismo tiempo, no significa que permanezcan residiendo o trabajando en el campo. Un aspecto colateral a la permanencia por varias generaciones es el empobrecimiento y deterioro de la infraestructura evidentes en algunos establecimientos por falta de manutención. La mayoría de las propiedades ha sufrido divisiones a partir de su tamaño original, de entre 4,000 y 60,000 ha antes de ser fraccionadas.

\section{Actividad turística en las propiedades}

Seis de estas propiedades inició sus actividades entre 1993 y 1995. Sin embargo, cabe resaltar, como se observa en el cuadro 1 , que cuatro de ellas seguía con la actividad turística en 2005. De las que cerraron, tres lo hicieron de manera permanente; en dos de los casos hubo venta de la propiedad. El cuadro 2 muestra los principales motivos para el cierre de las actividades turísticas, entre los que destaca en 2002 la poca rentabilidad y la deficiente preparación de la mano de obra, y en 2005, el interés por dedicarse a otras actividades profesionales. En el cuadro 3 se observan las dificultades para permanecer en la actividad turística. En 2002, la mitad de los propietarios mencionan la falta de promoción, mientras que en 2005 se manifiesta el nivel de exigencia de mano de obra calificada. En relación con la dificultad de acceso 


\section{Cuadro 1}

\section{Actividad turística en las propiedades}

\begin{tabular}{lccc}
\hline Número de fincas & 1997 & 2002 & 2005 \\
\hline Activas & 9 & 4 & 4 \\
Inactivas & - & 5 & 5 \\
Situación operacional & - & 1 & - \\
Cierre temporal & - & 3 & 3 \\
Cierre permanente & - & 1 & 2 \\
Venta & - & \\
\hline
\end{tabular}

\section{Cuadro 2}

Motivos para el cierre de la actividad turística

\begin{tabular}{lcc}
\hline & 2002 & 2005 \\
\hline Poco flujo de turistas & 1 & - \\
Poca rentabilidad & 2 & - \\
Poca preparación de los recursos humanos & 1 & - \\
Estacionalidad del turismo & 1 & - \\
Otras actividades profesionales & 1 & 2 \\
Problemas personales & 1 & 2 \\
Problemas familiares & 1 & 1 \\
Problemas de salud & - & 2 \\
No respondió & 1 & 1 \\
Base & 5 & 5 \\
\hline
\end{tabular}

Cuadro 3

Dificultades para permanecer en la actividad turística

\begin{tabular}{lccc}
\hline & 1997 & 2002 & 2005 \\
\hline Falta de divulgación & - & 1 & - \\
Falta de mano de obra calificada & - & - & 2 \\
Políticas desfavorables & 1 & - & - \\
Falta de servicios & 1 & - & - \\
Falta de incentivos & - & - & 1 \\
No ve dificultades & 5 & 2 & - \\
No respondió & 2 & 1 & 1 \\
Base & 9 & 4 & 4 \\
\hline
\end{tabular}

a estos sitios, Martínez y Monzonís (s.f.) refieren, a partir de datos de la OCDE, que en el contexto europeo cerca de $68 \%$ de las personas que utilizan los servicios de turismo rural se trasladan en auto, lo que refleja la importancia de la conservación adecuada de los caminos vecinales (Cánoves et al., 2004). Esta situación es similar en las fincas estudiadas, pues se relaciona, según estos autores, a las propias características de la actividad y al 
perfil de los turistas. En cuanto a la comunicación -segunda dificultad mas citada-, de acuerdo con Dóris Ruschmann (1998), ésta es fundamental para que las personas compren un determinado producto o utilicen un servicio. De acuerdo con Zimmermann (1996), en el caso del turismo rural la comunicación debe incluir esfuerzos tanto de los gobiernos municipales como de los propietarios.

En cuanto al potencial receptivo de las propiedades de acuerdo con el tipo de atención, la mayor parte de las fincas activas recibe turistas para pasar el día y pernoctar (75\%), incluyendo alimentación completa. Las demás sólo reciben visitantes para pasar el día e incluyen alimentación. Esto revela que la mayoría de las propiedades tienen una estructura de hospedaje mayor que aquéllas que reciben solamente para pasar el día.

Según los propietarios, el perfil de la mayor parte de los turistas se encuentra entre los 35 y 52 años, son profesionales libres, casados y viajan con sus hijos, originarios de diversas localidades del estado. Entre los servicios que se ofrecen en estos sitios se encuentran los paseos a caballo, 100\% de los huéspedes lo ve como el entretenimiento más atractivo; los trabajos del campo, que podrían constituir otro aspecto importante, sólo 25\% de los paseantes lo mencionaron, tal vez por la incompatibilidad física del habitante urbano con esas actividades o bien por desconocimiento de las mismas e, incluso, por la baja promoción por parte de los propietarios.

En cuanto a lo que opinan los turistas acerca de sus preferencias, según Mário Beni (1998), para éstos es más atractivo el manejo de ganado, la colocación de cercas, los galpones (almacenes para máquinas e implementos), paseos por los caminos, etc. Esto coincide con lo que informan Aliza Fleischer y Anat Tchetchik (2005) en Israel, donde las actividades agropecuarias no son atractivas para los turistas que visitan las fincas. También puede explicarse por lo que refieren Joaquim Almeida y Marcelino de Souza (2002) en cuanto a que el turismo rural busca proporcionar descanso y contacto con los valores culturales y patrimoniales tradicionales en un contexto diferente al de la ciudad.

Los huéspedes que permanecen de una a dos noches representa $50 \%$ y $25 \%$ lo hace por tres o cuatro noches. Esto sugiere varias posibilidades: el turista puede utilizar el lugar para hospedarse en el trayecto de un viaje más largo, o bien, es posible que crea que no hay mucho que hacer en una hacienda. Éste es uno de los aspectos que menciona Zimmermann (1996), pues esta 
práctica puede representar una mayor rentabilidad ya que es posible aprovechar los cuartos para transformar la vivienda rural en una posada. Otra modalidad que menciona el mismo autor es la de recibir turistas para pasar el día, en la cual ganan con el valor de los alimentos y la venta de productos.

En cuanto al retorno a la propiedad, la mayoría (75\%) de los huéspedes regresa de una a dos veces al año. Este dato sugiere una preferencia por salidas cortas al campo varias veces al año, en lugar de estancias prolongadas. Esta tendencia hacia vacaciones cortas más frecuentes parece ser una preferencia del mundo actual, donde el estrés diario se acumula rápidamente y son necesarios varios descansos a lo largo del año. Rosa María Yagüe (2002) observó una situación similar en España, lo que puede estar relacionado con la distancia en el tiempo de los periodos vacacionales y los fines de semana largos. En general, los propietarios atribuyen el retorno de los huéspedes a la atención, la ubicación, los atractivos, el contacto con la naturaleza-campo, el descanso-fuga del estrés, entre otros.

También existe la posibilidad de realizar actividades de pesca en estas propiedades, pues todas tienen autorización debido a la presencia de estanques que se usan en la agricultura y como abrevaderos. La cacería de aves, sin embargo, está bastante restringida y actualmente ninguna de las fincas tiene autorización para realizarla debido a una fuerte fiscalización estatal.

En cuanto a la atención, la mayoría de los huéspedes la consideran buena o excelente (75\%). Según Biagio Avena (2002), quien recibe huéspedes no vende solamente un producto material al cliente, pues es importante que se incluyan la amabilidad, el calor humano, el reconocimiento, la hospitalidad y el cuidado. En relación con la localización, predominó el hecho de que al turista le guste el lugar o región (25\%), así como el contacto con la naturaleza.

Los principales motivos de los propietarios para permanecer en la actividad turística son el hecho de que lo disfrutan (50\%) y el éxito de la empresa (50\%), además de que el sector de servicios ya forma parte de la vida en el medio rural (25\%).

En cuanto al equipamiento para el funcionamiento de la actividad turística, en 2002 la mayor inversión fue en infraestructura (100\%), con una baja en 2005 (75\%), principalmente en reformas y mejoras de la casa. En segundo lugar aparece la inversión en la compra de muebles y utensilios, que pasó de 33\% en 1997 a $50 \%$ en 2002 y $75 \%$ en 2005 . 


\section{Cuadro 4}

Calificación de los resultados

\begin{tabular}{lccc}
\hline & 1997 & 2002 & 2005 \\
\hline Excelentes & 3 & - & - \\
Buenos & 1 & - & 3 \\
Satisfactorios & - & - & 1 \\
Más positivos que negativos & - & 1 & - \\
Regulares, con tendencia a mejorar & & & \\
a mediano y largo plazos & - & 1 & - \\
Permiten continuar & - & 1 & - \\
No respondió & 5 & 1 & \\
Base & 9 & 4 & 4 \\
\hline
\end{tabular}

En el cuadro 4 se describe la percepción del propietario en cuanto a los efectos de la actividad turística en la propiedad. En 2005 gran parte de ellos (75\%) percibió los resultados como buenos y una menor proporción (25\%) como satisfactorios. Como es obvio, las respuestas sólo incluyen a los propietarios de las fincas que permanecen abiertas al turismo.

En cuanto al tipo de actividad turística, se constató que predomina del agroturismo sobre el turismo rural, pasando de 100\% de las fincas en 1997 a 75\% en 2002 y 2005. Esto indica que la actividad permanece como una fuente de ingresos complementaria a la actividad agropecuaria.

\section{Actividad primaria en las propiedades}

La producción de arroz, soya y maíz requiere de grandes inversiones como fertilizantes, semillas, plaguicidas, tractores y cosechadoras, entre otros. El maíz fue el producto más sembrado, posiblemente porque es un producto de fácil laboreo. Los cultivos de subsistencia como el frijol y la papa dulce casi no aparecen, ya que al propietario le resulta más barato comprarlos que cultivarlos. Los datos revelan que 38\% de los propietarios no siembra y sólo $25 \%$ lo hace por cuenta propia, mientras que aparceros $(75 \%)$ y arrendatarios $(7 \%)$ realizan la mayor parte de la actividad agrícola. En cuanto a la maquinaria, se observa un cambio complejo: en 1997 todos los propietarios poseían al menos un tractor, mientras que para 2005 sólo $25 \%$ de ellos lo tienen y ninguno cuenta con cosechadoras.

Todos los productores depositan sus productos en cooperativas de la región. La principal actividad ganadera continúa siendo 
Figura I

Generación promedio de empleos por finca

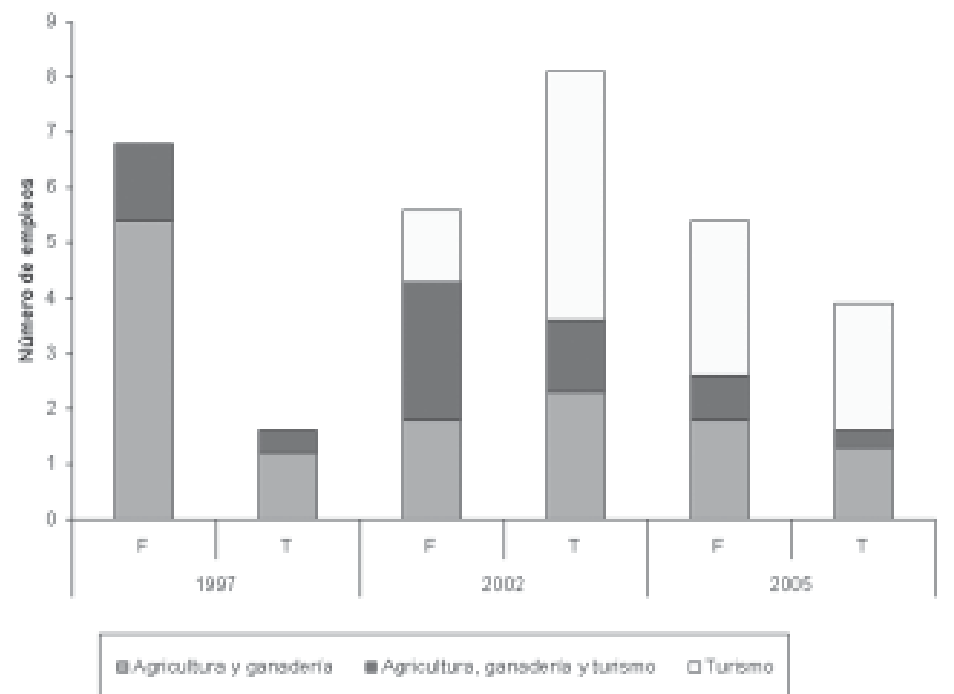

F: fijos, T: temporales.

Fuente: Investigación de campo (1997, 2002 y 2005).

\section{Cuadro 5}

Distribución porcentual de la renta

\begin{tabular}{cccc}
\hline Sector & 1997 & 2002 & 2005 \\
\hline Primario & 76 & 74 & 72 \\
Terciario & 24 & 26 & 28 \\
\hline
\end{tabular}

la bovinocultura de carne y de cría (75\%), así como la de leche para consumo interno en su totalidad (100\%).

\section{Generación de empleo y renta por sector}

En la figura se muestra la evolución en la generación de empleos, donde se observa un aumento en el número de empleos generados por finca entre 1997 y 2002 y una disminución para el periodo de 2002 a 2005 . El notable aumento de empleos temporales en 2002 se puede atribuir a una situación macroeconómica favorable en ese momento. Sin embargo, el número de empleos generados exclusivamente por la actividad turística mostró un 
repunte importante en 2005, ocupando ahora más personas en el sector terciario que en el primario, pero también se observa una disminución en empleos multifuncionales. Por otra parte, el porcentaje de renta proveniente del turismo también experimentó un ligero aumento, pasó de $24 \%$ de los ingresos totales en 1997 a $26 \%$ en 2002 y a $28 \%$ en 2005, lo que refleja la viabilidad económica de la actividad turística y su capacidad generadora de empleos.

\section{Conclusiones}

Muchos de los propietarios viven exclusivamente de los rendimientos obtenidos en el campo, lo que requiere mucha habilidad debido a las dificultades derivadas de la actividad primaria. Sin embargo, más de la mitad de ellos tiene otras actividades, lo que los lleva a no residir exclusivamente en la propiedad. De cualquier modo, en ambos casos la actividad turística constituye una renta complementaria sólo para quienes practican el agroturismo, el cual, de acuerdo con Mário Beni (1998), provee una renta complementaria a la actividad primaria.

Las propiedades las administran los dueños de éstas, lo cual indica un apego a la vida rural. En general, la idea de iniciar la actividad turística en la finca fue de ellos mismos, revelando un espíritu emprendedor. Sin embargo, no se percibe un interés de los hijos por hacerse cargo de las fincas y la mayoría de ellos tiene estudios en áreas no vinculadas al sector rural.

Se constató que en la región predomina el agroturismo sobre el turismo rural, pues la mayoría de los propietarios rurales ve la actividad turística como complementaria, aunque también hay quienes la realizan como fuente principal de renta de la propiedad. Dentro de los motivos de los propietarios para practicar estas actividades destacan los económicos, principalmente el deseo de aumentar la rentabilidad y agregar valor, lo que coincide con estudios anteriores (Beni, 1998; Tulik, 1997).

En cuanto a los motivos de los propietarios para continuar, las razones más mencionadas son el hecho de creer en la actividad turística como fuente de renta adicional a largo plazo, así como el deseo de convivir con personas diferentes.

Sólo cuatro de las nueve fincas siguen con la actividad turística. La principal dificultad para continuar es la estacionalidad del turismo. Eurico Santos (1998) también menciona la falta de mano de obra especializada, la ausencia de promoción y el hecho de 
tener que abrir la casa al público. Sin embargo, la mayoría de los propietarios que siguen con esta actividad percibe los resultados como buenos y satisfactorios, y una pequeña parte como excelentes. En todas las fincas se ha invertido en infraestructura (mantenimiento y mejora de las casas).

Casi todas las propiedades activas reciben huéspedes para pasar el día y para pernoctar, incluyendo los alimentos. La mayoría de ellos permanece de una a dos noches y regresan de una a dos veces al año, debido principalmente a la calidad de la atención recibida. El factor que motiva la práctica del agroturismo y del turismo rural es el contacto con la naturaleza, así como el silencio y la tranquilidad del campo como contraparte al estrés de la vida urbana.

En síntesis, el estudio sugiere que el hombre del campo continúa en él, sin abandonar la actividad agropecuaria, y que el turismo representa su inserción en la pluriactividad. Si bien algunas de las fincas no han sido exitosas, pensamos que la actividad se puede trabajar mejor y que es necesaria una mayor difusión para que se expanda y aumente la calidad del producto que se ofrece. Los resultados de este estudio contribuyen para un mayor conocimiento de la actividad turística en la región a través de la percepción de los propietarios rurales. Esperamos abordar en estudios posteriores las características de la demanda desde la perspectiva del turista.

\section{Bibliografía}

Almeida, Joaquim y Marcelino de Souza (2002), Conceitualizaçãio do rutismo no espaço rural" en < http://www.ufsm.br/turismorural >, 16 de noviembre de 2002.

ATAR (Associazione per il Turismo In Ambiente Rurale) (1984), Vademecum dell'operatore di agroturismo, Roma, Terranostra.

Avena, Biagio (2004), “Acolhimento de Qualidade. Fator diferenciador para o incremento do turismo en la sociedade posindustrial”, en <http:/www.abbtur.com.br/CONTEUDO/ trabalhos/trab11.htm>, 2 de mayo de 2004.

Bardin, Laurence (1977), Análise de conteúdo, Sao Paulo, Brasil, Martins Fontes. 
Beni, Mário (1998), "Análise estrutural do turismo", Turismo Visão e Ação, año 2, 4, Sao Paulo, Brasil, pp. 80.

Campanhola, Clayton y José Graziano da Silva (2003), O novo rural brasileiro, uma análise estadual sul, sudeste e centrooeste, vol. 3, São Paulo, Brasil, Embrapa Meio Ambiente.

Carneiro, Maria José y Renato Maluf (eds.) (2003), Para alem da produção: multifuncionalidade e agricultura familiar, Río de Janeiro, Brasil, Mauad.

Cánoves, Gemma, Montserrat Villarino, Gerda Priestley y Asunción Blanco (2004), "Rural tourism in Spain: an analysis of recent evolution”, Geoforum, 35(6), Elsevier, Ámsterdam, pp. 755-769.

Engevix (1997), Plano de reestruturação econômica para a metade sul do Rio Grande do Sul. Relatório Final, Porto Alegre, Brasil, Engevix.

Fleischer, Aliza y Anat Tchetchik (2005), "Does rural tourism benefit from agriculture?", Tourism Management, 26(4), Elsevier, Ámsterdam, pp. 493-501.

Martínez, Francisco Juan y Javier Solsona Monzonís (s.f.), Alojamiento turístico rural: gestión y comercialización, Madrid, España, Síntesis.

Martínez-Figueroa, Luis Andrés (2006), “Turismo rural: una nueva alternativa de agronegocios", en <http://www.promer.cl/ getdoc.php?docid=344>, 20 de mayo de 2006.

Oliveira, Cássio Garkalns de Souza (2001), “O sucesso como posible fator de descaracterização de empreendimentos de turismo rural”, en Cássio Garkalns de Souza Oliveira, José Carlos de Moura y Marcos Sgai (eds.), Turismo no espaço rural brasileiro. Anais do $3^{\circ}$ Congresso Brasileiro de Turismo Rural, Piracicaba, Fealq, pp. 277-283.

Riveros, Hernando y Marvin Blanco (2003), "El agroturismo, una alternativa para revalorizar la agroindustria rural 
como mecanismo de desarrollo local", documento técnico, Lima, Perú, IICA-Prodar.

Ruschmann, Dóris (1998), "O turismo rural e desenvolvimento sustentable”, en Joaquim Almeida, José Froehlich y Mário Riedl (eds.), Turismo rural e desenvolvimento sustentable, Santa Maria, Brasil, CNPq-CAPES.

Santos, Eurico de Oliveira (1998), Turismo rural en fazendas con mas de 1000 ha no estado do Río Grande do Sul, monografía de especialización en gestión y producción del turismo, Río Grande do Sul, Pontificia Universidade Católica do Río Grande do Sul-Famecos, Brasil.

Seltiz, Claire, Lawrence Wrightsman y Stuart Cook (1987), Métodos de pesquisa nas relações sociais. V. Delineamentos de pesquisa, Sao Paulo, Brasil, McGraw-Hill.

Setur (Secretaria de Turismo do Estado do Rio Grande do Sul) (1997), "Lista das propriedades rurais do Rio Grande do Sul”, en <www.setur.gov.rs.br>, 5 de septiembre de 1997.

Tulik, Olga (1997), "Do conceito a las estratégias para o desenvolvimento do turismo rural", en A. B. Rodrigues (ed.), Turismo: desenvolvimento local, Sao Paulo, Huccitec, pp. 136-143.

Tulik, Olga (2003), Turismo rural. Coleção ABC do turismo, Sao Paulo, Brasil, Aleph.

Veiga, José Eli (2002), Ciudades imaginárias: o Brasil é menos urbano do que se calcula, Campinas, Editora Autores Associados.

Yagüe Perales, Rosa María (2002), "Rural tourism in Spain”, Annals of Tourism Research, 29(4), Elsevier, Ámsterdam, pp. 1101-1110.

Zdepski, Fabíola Bevervanço (2001), “Aspectos determinantes da oferta para o desenvolvimento do turismo rural", en, Cássio Garkalns de Souza Oliveira, José Carlos de Moura y Marcos Sgai (eds.), Turismo no espaço rural brasileiro. 
Anais do $3^{\circ}$ Congresso Brasileiro de Turismo Rural, Piracicaba, Fealq, pp. 155-160.

Zimmermann, Adonis (1996), Turismo rural: un modelo brasileiro, Florianópolis, edición del autor.

Recibido: 22 de marzo de 2006. Reenviado: 14 de mayo de 2007. Aceptado: 20 de junio de 2007.

Eurico de Oliveira Santos. Es maestro en extensión rural por la Universidad Federal de Santa María, Brasil. Actualmente se encuentra adscrito al Centro de Investigación en Ciencias Agropecuarias de la Universidad Autónoma del Estado de México y cursa el doctorado en ciencias agropecuarias y recursos naturales en la misma universidad.

Carlos Ernesto González Esquivel. Es doctor en filosofía y agroecología por la Universidad de Londres. Actualmente se encuentra adscrito al Centro de Investigación en Ciencias Agropecuarias de la Universidad Autónoma del Estado de México. 